\title{
TIME SERIES OF SAR IMAGE FRACTAL MAPS
}

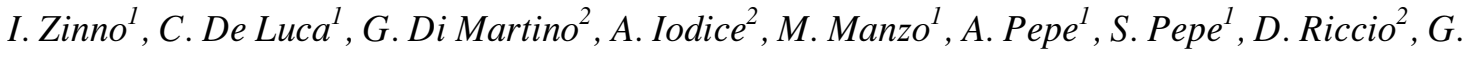 \\ Ruello $^{2}$, E. Sansosti ${ }^{1}, P$. Tizzani $^{1}$
}

(1) Istituto per il Rilevamento Elettromagnetico dell'Ambiente (IREA), CNR, Napoli, Italy

(2) Università di Napoli Federico II - DIETI, Napoli, Italy

\begin{abstract}
In this paper the analysis of time series of fractal dimension maps generated from multi-pass SAR images is dealt with. The objective is twofold: on the one hand such an analysis is addressed to assess the performance of the algorithm for the fractal dimension estimation from a single SAR image, i.e., to verify the fractal estimation efficiency on natural scenes within the multiple sensor passes. On the other hand, by exploiting the statistics evaluated starting from the fractal time series, a final fractal dimension map, including only the areas in which the fractal estimation is efficient and strongly denoised from the speckle effect, is obtained. The analysis has been performed on a Cosmo-SkyMed data-set of 42 stripmap images spanning the time period from October 2009 to December 2012, acquired over the SommaVesuvius volcanic complex (South Italy), which is in a quiescent stage since the last eruption occurred in 1944.
\end{abstract}

Index Terms - SAR, fractal dimension, SAR image time series

\section{INTRODUCTION}

It is widely recognized in literature that the fractal dimension is a significant geophysical parameter describing natural surfaces [1], [2]; it represents the distribution of the roughness over different spatial scales [1], [2] and, in case of volcanic structures, it has been related to the specific nature of materials and to the effects of active geodynamic processes [3]. Fractal surfaces have a fractal dimension $D$ included in the ]2,3[ interval; in particular, for natural surfaces $D$ is included in ]2,2.5[ interval, see [1].

Several works have been presented in literature [1], [4]-[10], aimed at evaluating the electromagnetic field backscattered by natural surfaces starting from the knowledge of their fractal parameters. On the other side, it has recently been presented an algorithm [11] that, starting from a single amplitude Synthetic Aperture Radar (SAR) image (which is related to the electromagnetic field backscattered by the illuminated scene), allows the computation of the fractal dimension map of the observed area. Furthermore, the fractal dimension of natural areas, estimated from the corresponding SAR images, proved to be very stable as the sensor illumination conditions and viewing geometry change, which is an uncommon feature of parameter extraction from SAR images [12], [16].

In this paper time series of fractal maps generated from multi-pass SAR images are considered. Our analysis is twofold; on the one hand, it is aimed at the performance assessment of the algorithm for fractal dimension extraction from a single SAR image, thus verifying the stability of the estimator for stable natural scenes within the multiple sensor passes. On the other hand, we jointly exploit the mean and standard deviation maps of the fractal dimension evaluated by exploiting the time series of the fractal maps; the averaging operation is done, pixel by pixel, over the time variable, after a proper co-registration of the fractal maps. The result is a final fractal map that includes only the areas where the $D$ estimation is considered reliable and stable (i.e., whose standard deviation computed over the time series is reasonably small). It is clear that the resulting map is strongly improved, as the average operation reduces the noise content due to the speckle, which affects the SAR images used to retrieve each fractal map. As a matter of fact, the fractal dimension is retrieved from the SAR image local spectrum, whose estimation (properly performed through the Capon estimator [13]), mainly at high frequencies, i.e., in correspondence to small spatial scales, is influenced by the speckle effect present on the SAR image. The analysis is performed on a Cosmo-SkyMed (CSK) data-set of 42 stripmap images spanning the time period from October 2009 to December 2012, acquired over the SommaVesuvius volcanic complex (South Italy), which is in a quiescent stage since the last eruption occurred in 1944.

The retrieved time series of the fractal dimension values show that, over stable natural areas, the fractal dimension values do not appreciably change (as also confirmed by the 


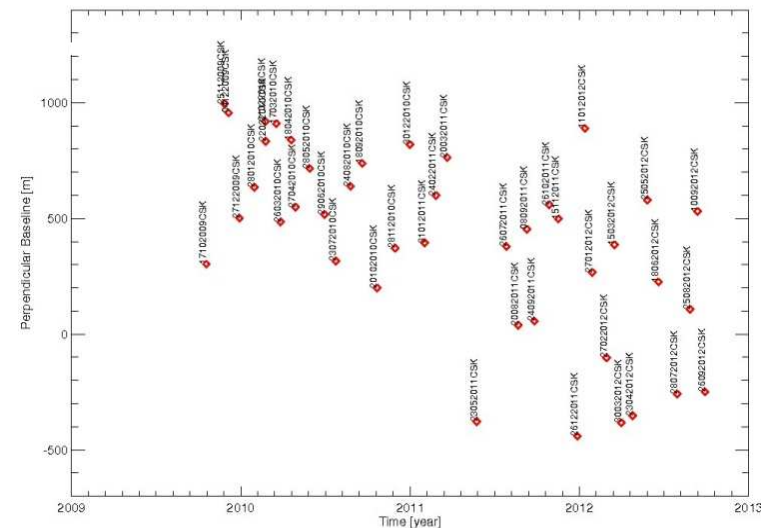

Fig. 1: Representation in the temporal/perpendicular baseline plane of the employed SAR acquisitions.

small values of the associated temporal standard deviation), although they can be retrieved from SAR images acquired at different times and orbital positions, thus stating the goodness of the fractal algorithm performance.

\section{FRACTAL DIMENSION ESTIMATION}

The fractal dimension computation is performed through a spectral analysis of the SAR amplitude data, whose details are presented in [11]. In particular, dealing with power-law spectra, an appropriate spectral estimator, that minimizes high variance and leakage problems, is used: the Capon estimator [13].

Furthermore, in order to obtain a point-by-point map of the fractal dimension, the spectral estimation is performed using a sliding window that encloses small portions of the image that are assumed to have the same fractal parameters; as a consequence, a unique averaged value of the fractal dimension is assigned to the center of the window. The dimension of the sliding window results from a trade-off between the accuracy of the estimation and the resolution required for the generated fractal maps.

\section{FRACTAL MAP TIME SERIES}

The main objective of this paper is the analysis of the performance of the technique described in the previous section. Such an analysis has been conducted considering a time series of SAR data acquired by the Cosmo-SkyMed constellation sensors over the Somma-Vesuvius volcanic complex (South Italy). The SAR data-set consists of 42 images collected through ascending passes in stripmap mode and spanning the time period from October 2009 to December 2012. Those images are acquired at a sidelooking angle of about $44^{\circ}$, at different orbital position, and present a spatial resolution of about $3 \mathrm{~m} \times 3 \mathrm{~m}$. In Figure 1 the distribution of the exploited SAR acquisitions in the temporal/perpendicular baseline plane is shown.

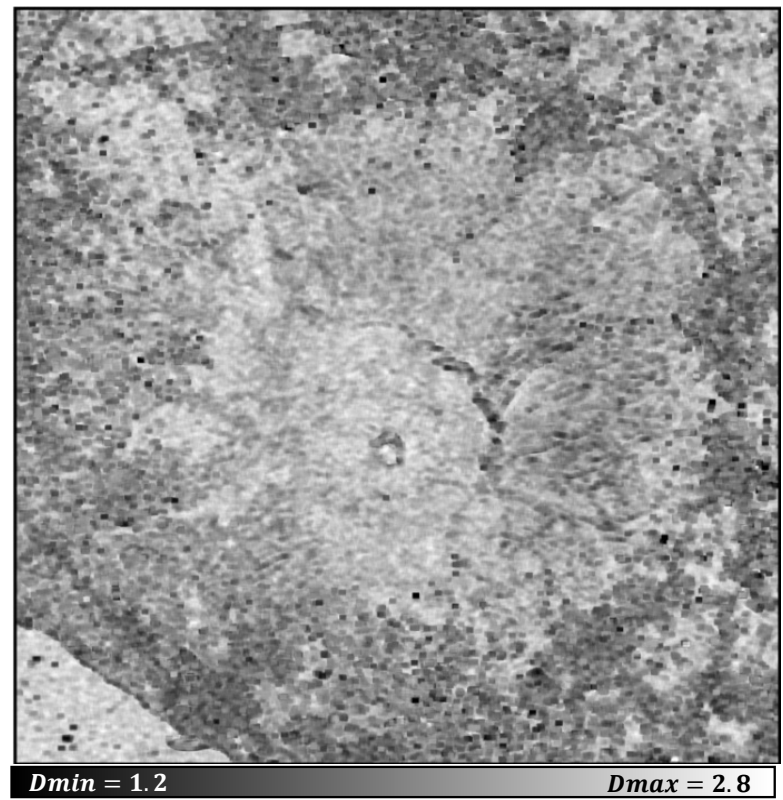

Fig. 2: Fractal dimension map generated from a single SAR image

Starting from the available SAR amplitude images, we generated the corresponding fractal dimension maps that were subsequently co-registered [14] with respect to the (fractal) map relevant to one reference image (i.e., the master image). The co-registration operation has been performed directly on the fractal maps, rather than on the starting SAR images, to avoid the introduction of artifacts that could significantly alter the fractal dimension estimate.

Then, we implemented a three-dimensional stack composed by the fractal maps ordered according to the acquisition date. Starting from the computed stack of fractal maps, we evaluated (pixel by pixel) both the average and the standard deviation fractal dimension maps, the averaging operation being carried out over time. As clearly shown in Figures 2 and 3 , the mean fractal dimension map (Figures 3) is significantly less noisy than that generated from a single SAR image (Figures 2). As a matter of fact, the average operation reduces the noise artifacts due to the speckle, which affects the SAR images used for the single fractal map computation.

In Figure 3 the resulting mean fractal dimension map is depicted; it ranges in the $[1.30,2.74]$ interval; the areas where the fractal dimension is lower than 2, which correspond to the darker regions of the map, are associated to the presence of man-made objects or layover zones that are clearly detected as non-fractal objects.

Moreover, in Figure 4, the map of the fractal dimension standard deviation values, along the considered temporal axis, is presented. The standard deviation values are in the $[0.016,0.132]$ range; however, all the natural areas (i.e., the volcanic cone and slopes) present a very small standard deviation (values lower than 0.05 , corresponding to the 


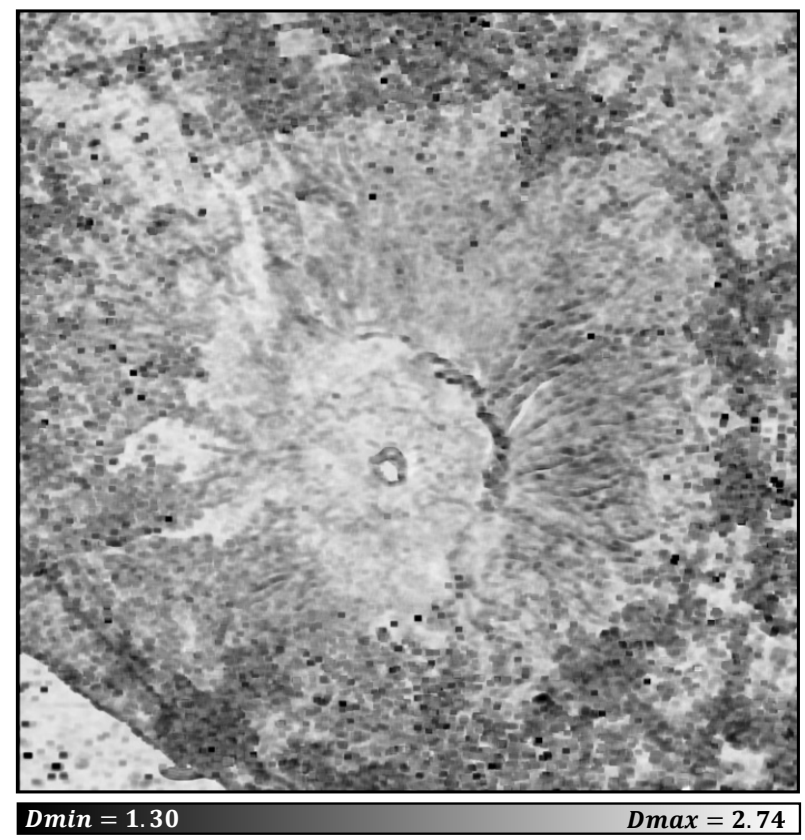

Fig. 3: Map of the fractal dimension mean values evaluated as from the stack of 42 co-registered fractal maps.

darker regions of the standard deviation map), while in the urban context the standard deviation gets to values of one order of magnitude larger.

This result confirms both the validity of the model and the efficiency of the estimation algorithm over natural areas. It is worth noting that the fractal estimation technique is based on a theoretical model that is valid only for natural surfaces; hence, although it is able to detect non fractal object providing a fractal dimension value outside the range of fractality ]2,3[, over urban areas it is not stable. In Figure 5, the fractal map obtained by jointly exploiting the mean and the standard deviation maps ( threshold value $=0.05$ ) is presented. In this map the areas masked with black color are those in which either the model is not valid or the estimation algorithm is not stable, while in the remaining areas (in color) the $D$ estimation is reliable and stable.

As it can be appreciated from the fractal map in Figure 5 the fractal dimension, describing the roughness of natural surfaces, is able to distinguish different geomorphologic/ vegetation properties of the observed scene. It has been studied that the most recent volcanic lava flows exhibit a fractal dimension lower than that of the oldest- depositional processes, often covered by vegetation [15].

The time-series of the pixel by pixel estimated fractal dimension values show that, over invariant natural areas, the fractal dimension values do not show significant changes; on the contrary, over urban areas, it correctly assumes values outside the natural surfaces fractality range and show strong fluctuations. In particular, in Figures 6 and 7, the

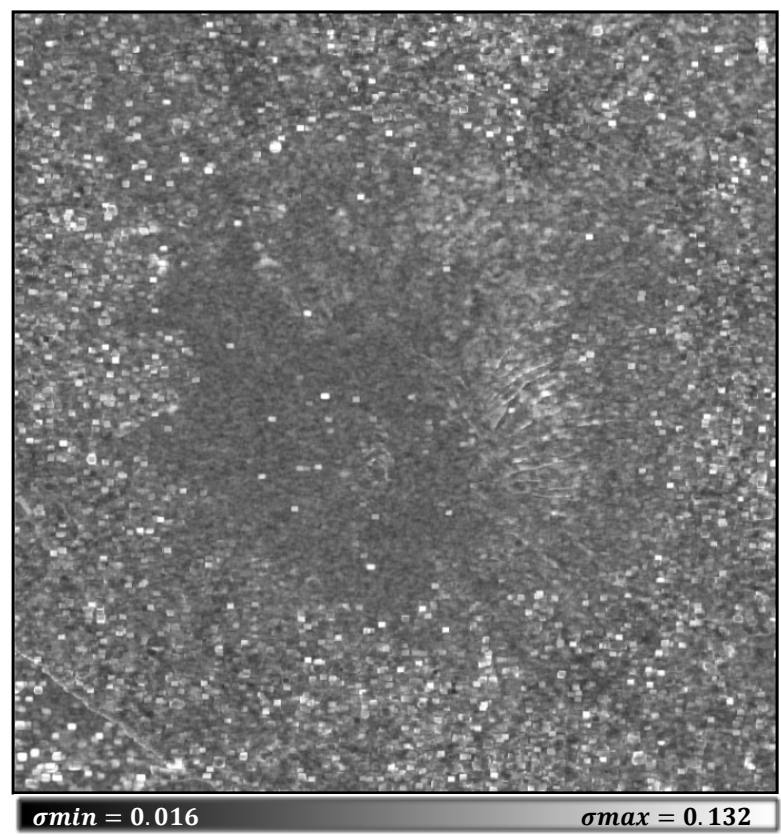

Fig. 4: Map of the fractal dimension standard deviation values evaluated as from the stack of 42 co-registered fractal maps.

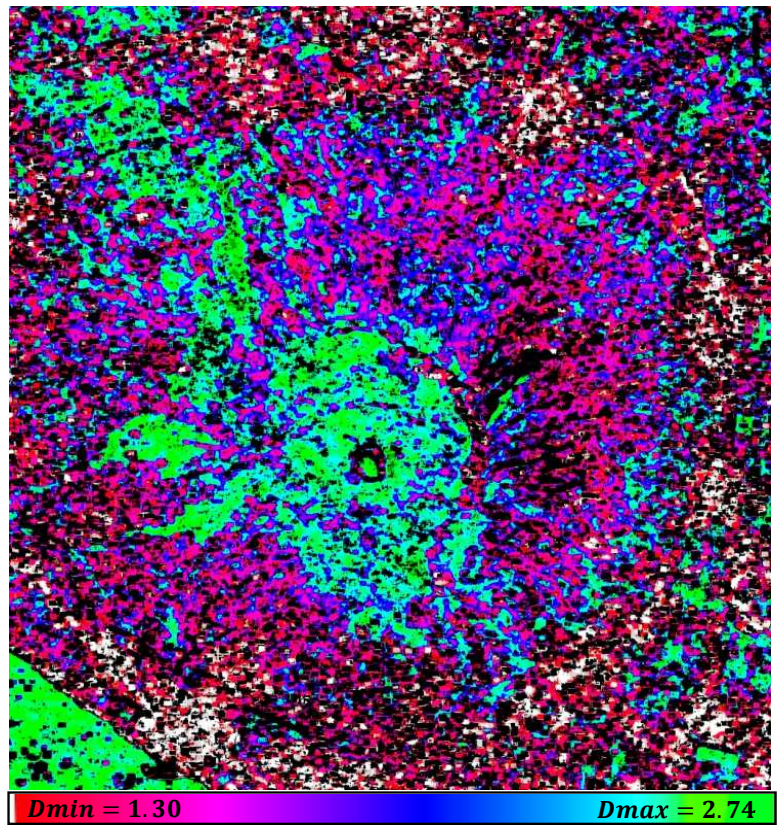

Fig. 5: Fractal map that includes only the areas in which the $D$ estimation is considered efficient and stable (in color), and that masks out other areas with the black color.

behaviors of the fractal dimension estimated in two pixels with respect to the temporal axis in a natural and an urban area, respectively, are shown. The first considered pixel 


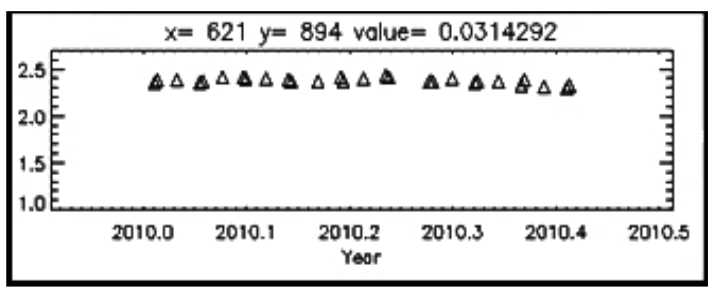

Fig. 6: Behavior of a the fractal dimension $D$ of a pixel located in a natural area with respect to the acquisition times. On the top, the standard deviation value is also reported.

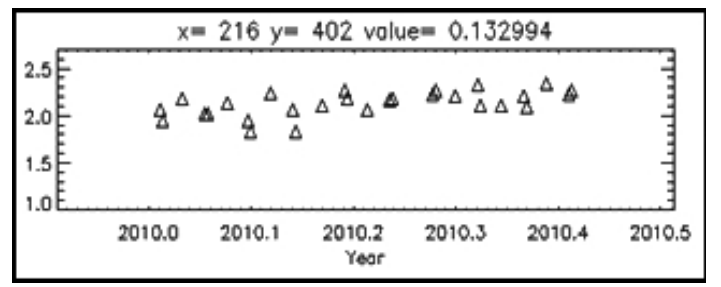

Fig. 7: Behavior of a the fractal dimension $D$ of a pixel located in an urban area with respect to the acquisition times. On the top, the standard deviation value is also reported.

belongs to the left (with respect to Fig. 5) side of the Vesuvius volcano, it presents a mean fractal dimension equal to 2.36 and a standard deviation equal to 0.03 and it exhibits a definitely homogeneous behavior.

On the contrary, the pixel belonging to the urban area, with a mean fractal dimension equal to 2.18 , shows a strongly fluctuating behavior as represented by the standard deviation equal to 0.13 . It is worth noting that in this latter case, the mean estimated value of the fractal dimension falls in the fractality range of natural surfaces, although this is not the case. This stresses the utility of exploiting a mask that automatically discards points for which the estimated fractal values are not reliable.

\section{CONCLUSION}

In this paper time series of fractal maps generated from multi-pass SAR images are considered. Our analysis is aimed, on the one hand, at assessing the stability of the $D$ estimation on natural scenes that do not vary in multi-pass SAR images. On the other hand, we jointly exploit the maps of fractal dimension mean and standard deviation value obtained evaluating these statistics, pixel by pixel, over the time series of fractal maps properly co-registered.

The analysis has been performed on a Cosmo-SkyMed dataset of 42 stripmap images spanning the time period from October 2009 to December 2012, acquired over the SommaVesuvius volcanic complex (South Italy), which is in a quiescent stage since the last eruption occurred in 1944.

The result is a final fractal map that includes only the areas in which the $D$ estimation is considered reliable and stable.
Moreover, the fractal dimension values estimated over the natural areas result strongly improved: the average operation significantly reduces the noise affecting the single fractal map that is due to the speckle phenomenon typical of a single SAR image.

\section{REFERENCES}

[1] G. Franceschetti, D. Riccio, Scattering, Natural Surfaces and Fractals. Academic Press, Burlington (MA), USA, 2007.

[2] B.B. Mandelbrot, The Fractal Geometry of Nature, New York: W.H.Freeman \& C., 1983

[3] D.L. Turcotte, Fractals and Chaos in Geology and Geophysics (second edition), Cambridge University Press, 1997.

[4] O.Y. Yordanov and K. Ivanova, "Kirchhoff Diffractals", J. Phys. A Math. Gen., 27, pp.5979-5993, 1994.

[5] T. Elfouhaily, D. R. Thompson, D. Vandemark, and B. Chapron, "A new bistatic model for electromagnetic scattering from perfectly conducting random surfaces," Waves Random Media, vol. 9, pp. 281-294, 1999.

[6] G. Franceschetti, A. Iodice, D. Riccio, "Fractal models for scattering from natural surfaces" in Scattering, edited by R.Pike and P.Sabatier, London: Academic Press, pp. 467-485, 2001.

[7] S. Perna, A.Iodice, "On the Use of Series Expansions for Kirchhoff Diffractals", IEEE Trans. Antennas Propagat, vol. 59, issue 2, pp.595-610, 2011.

[8] S.Perna, A. Iodice, "Asymptotic Behavior of Two Series Used for the Evaluation of Kirchhoff Diffractals," IEEE Trans. Antennas Propagat., vol. 59, Issue 6, pp. 2442-2444, 2011

[9] S.Perna, A.Iodice, "An Algorithm for Efficient and Effective Evaluation of Scattering from Fractal Surfaces," IEEE Trans. Geosci. Remote Sens.,vol. 50, Issue 9, pp.3554-3556, 2012.

[10] A.Iodice, A.Natale, D.Riccio, "Kirchhoff Scattering From Fractal and Classical Rough Surfaces: Physical Interpretation," IEEE Trans. Antennas Propagat.,vol. 61, Issue 4, 2013

[11] G. Di Martino, D. Riccio, I. Zinno, "SAR Imaging of Fractal Surfaces," IEEE Trans. Geosci. Remote Sens., vol.50, no.2, pp. 630-644, Feb. 2012.

[12] Di Martino, G.; Iodice, A.; Riccio, D.; Ruello, G.; Zinno, I., "Fractal maps dependence on SAR look angle," Geoscience and Remote Sensing Symposium (IGARSS), 2012 IEEE International, vol., no., pp.6641-6644, 22-27 July 2012.

[13] T. Austin, A. W. England, G. H. Wakefield, "Special problems in the estimation of power-law spectra as applied to topographical modeling”, IEEE Trans. Geosci. Remote Sens., vol. 32, no. 4, pp. 928- 939, July 1994.

[14] G. Franceschetti and R. Lanari, Synthetic Aperture Radar Processing. New York: CRC Press, 1999.

[15] De Luca et al., "Landscape roughness analysis of Mt. Etna volcanic complex detected viafractal geometry", EGU General Assembly 2013 Vol. 15, EGU2013-9294, 2013

[16] G. Di Martino, A. Iodice, D. Riccio, G. Ruello, and I. Zinno, "Angle Independence Properties of Fractal Dimension Maps Estimated from SAR Data", IEEE J. Sel. Topics Appl. Earth Observ., vol. 6, no. 3, June 2013. 